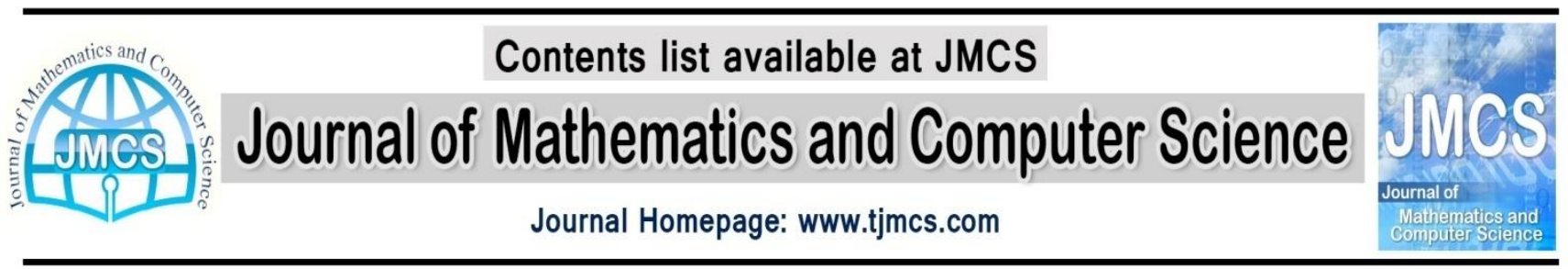

\title{
Some Results on the Generalized Rough Lie Subalgebras
}

Article history:

Received March 2013

Accepted October 2014

Available online October 2014

\author{
S. B. Hosseini ${ }^{1, a}$; A. Kazemi ${ }^{\text {b }}$
}

Department of Mathematics, Sari Branch, Islamic Azad University, Sari, Iran.

E-mail address: ${ }^{a}$ sbhosseini@iausari.ac.ir ; ${ }^{b}$ Arefe.kazemi65@gmail.com

\begin{abstract}
The main purpose of this paper is to introduce and discuss the concept of $T$-roughness in Lie subalgebra and generalized $T$-rough Lie subalgebras. We define a set-valued homomorphism on a Lie algebra and study some of their properties and useful applications.
\end{abstract}

Keywords: Lower approximation; Upper approximation; $T$-rough set; Set-valued homomorphism; Lie algebras.

\section{Introduction}

The notion of rough sets has been introduced by Z. Pawlak [11, 12], Z. Pawlak and A. Skowron [13] and T. Iwinski [8]. It soon invoked a natural question concerning a possible connection between rough sets and algebraic systems. The algebraic approach to rough sets have been studied by Z. Bonikowaski [2]. R. Biswas and S. Nanda [1] introduced the notion of rough subgroups. N. Kuroki [10] introduced the notion of rough ideals in a semigroup. B. Davvaz [4] introduced the notion of rough subring with respect to an ideal of a ring. O. Kazanci, B. Davvaz [9] discussed the structure on the rough prime (primary) ideals. In [15], W. Zhang, W. Wu considered some other results. B. Davvaz [3] introduced T- rough set and $T$ - rough homomorphism in a group. In [14], S. Yamak, O. Kazanci, B. Davvaz introduced the generalized lower and upper approximation in a ring. S. B. Hosseini et al. [6, 7] introduced $T$-rough ideal in a semigroup and in a commutative ring. The rough set theory is an extension of set theory, in which a subset of a universe is described by a pair of ordinary sets called the lower and the upper approximations. The lower approximation of a given set is the union of all the equivalence classes which are subsets of the set, and the upper approximation is the union of all the equivalence classes which have a non-empty

\footnotetext{
${ }^{1}$ Corresponding author.
} 
intersection with the set. The rough sets are a suitable mathematical model of vague concepts, i.e., concepts without sharp boundaries. In this paper, a set-valued homomorphism on a Lie algebra and the concept $T$-rough Lie subalgebra are introduced and some interesting properties are proved. Suppose that $U$ is a non-empty set. A partition or classification of $U$ is a family $\Theta$ of non-empty subsets of $U$ such that each element of $U$ is contained in exactly one element of $\Theta$. It is vitally important to recall that an equivalence relation $\theta$ on a set $U$ is a reflexive, symmetric and transitive binary relation on $U$. Each partition induces an equivalence relation on $U$. If $\theta$ is an equivalence relation on $U$, then for every $x \in U,[x]_{\theta}$ denotes the equivalence class of $\theta$ determined by $x$. For any $X \subseteq U$, we write $X^{c}$ to denote the complement of $X$ in $U$ that is the set $U \backslash X$. A pair $(U, \theta)$ where $U \neq \varnothing$ and $\theta$ is an equivalence relation on $U$ is called an approximation space. Let $P(U)$ be the set of all subsets of $U$ and for an approximation space $(U, \theta)$ by a rough approximation in $(U, \theta)$ we mean a mapping

$$
\text { Apr }: P(U) \rightarrow P(U) \times P(U) \text { defined by for every } X \in P(U), \operatorname{Apr}(X)=(\underline{A p r}(X), \overline{\operatorname{Apr}}(X))
$$

where

$$
\underline{\operatorname{Apr}}(X)=\left\{x \in U \mid[x]_{\theta} \subseteq X\right\} ; \overline{\operatorname{Apr}}(X)=\left\{x \in U \mid[x]_{\theta} \cap X \neq \varnothing\right\} .
$$

$\operatorname{Apr}(X)$ is called the lower rough approximation of $X$ in $(U, \theta)$ whereas $\overline{\operatorname{Apr}}(X)$ is called the upper rough approximation of $X$ in $(U, \theta)$.

Given an approximation space $(U, \theta)$ a pair $(A, B)$ in $P(U) \times P(U)$ is called a rough set in $(U, \theta)$ if $(A, B)=(\underline{A p r}(X), \overline{\operatorname{Apr}}(X))$ for some $X \in P(U)$.

\section{Set-valued Lie homomorphism and $T$-rough Lie subalgebra}

In this section, we define the concept of a set-valued Lie homomorphism and give some important examples of a set-valued mapping. We also investigate some basic properties of the generalized lower and upper approximation operators in a Lie algebra. We generalize the rough Lie subalgebra called $T$-rough Lie subalgebra. We apply the notion of $T$-rough sets in a Lie algebra and extend some theorems which have been proved in $[3,4,6,7]$.

Throughout in this section and the next, the set of all non-empty subsets of $Y$ is denoted by $P^{*}(Y)$.

Definition 2.1 [3] Let $X$ and $Y$ be two non-empty sets and $\varnothing \neq B \subseteq Y$. Let $T: X \rightarrow P^{*}(Y)$ be a setvalued mapping. The lower inverse and upper inverse of $B$ under $T$ are defined by

$$
L_{T}(B)=\{x \in X \mid T(x) \subseteq B\} ; U_{T}(B)=\{x \in X \mid T(x) \cap B \neq \varnothing\},
$$

respectively.

Definition 2.2 [3] Let $X$ and $Y$ be two non-empty sets and $B \in P^{*}(Y)$. Let $T: X \rightarrow P^{*}(Y)$ be a setvalued mapping. $\left(L_{T}(B), U_{T}(B)\right)$ is called a $T$ - rough set with respect to $B$. 
Proposition $2.3[3,6,7]$ Let $X$ and $Y$ be two non-empty sets and $A, B \subseteq Y$. Let $T: X \rightarrow P^{*}(Y)$ be a set-valued mapping, then the following holds:

(i) $U_{T}(A \cup B)=U_{T}(A) \bigcup U_{T}(B)$;

(ii) $L_{T}(A \cap B)=L_{T}(A) \bigcap L_{T}(B)$;

(iii) $A \subseteq B$ implies $L_{T}(A) \subseteq L_{T}(B)$ and $U_{T}(A) \subseteq U_{T}(B)$;

(iv) $L_{T}(A) \cup L_{T}(B) \subseteq L_{T}(A \cup B)$ and $U_{T}(A \cap B) \subseteq U_{T}(A) \cap U_{T}(B)$.

Example 2.4 (i) Let $(U, \theta)$ be an approximation space and $T: U \rightarrow P^{*}(U)$ be a set-valued mapping where $T(x)=[x]_{\theta}$, then for any $B \subseteq U, L_{T}(B)=\underline{A p r}(B)$ and $U_{T}(B)=\overline{A p r}(B)$. So, rough sets are $T$-rough sets. In fact, $T$-rough sets are a generalization of rough sets.

(ii) Let $\mathrm{Z}$ be integer numbers set and $T: Z \rightarrow P^{*}(\mathrm{Z})$ be a set-valued mapping where $T(n)=n Z$ for all $n \in Z$. If $A=2 Z$, then $L_{T}(A)=2 Z$ and $U_{T}(A)=Z$.

Definition 2.5 Let $F$ be a field. A Lie algebra over $F$ is an $F$-vector space $L$, together with a bilinear map, the Lie bracket

$$
L \times L \rightarrow L, \quad(x, y) \rightarrow[x, y]
$$

satisfying the following properties:

$\cdot[x, x]=0$ for all $x \in L$;

$\cdot[x,[y, z]]+[z,[x, y]]+[y,[z, x]]=0$ for all $x, y, z \in L$.

The Lie bracket $[x, y]$ is often referred to as the commutator of $x$ and $y$.

Condition (L2) is known as the Jacobi identity.

Definition 2.6 (i) If $L$ is a Lie algebra. We defined a Lie subalgebra of $L$ to be a vector subspace $\varnothing \neq K \subseteq L$ such that

$$
[x, y] \in K \text { for all } x, y \in K \text {. }
$$

(ii) If $A$ and $B$ be two Lie subalgebras of $L$ then we define $[A, B]$ as follows:

$$
[A, B]=\operatorname{span}\{[a, b] \mid a \in A, b \in B\}=\left\{\sum_{i=1}^{n} \lambda_{i}\left[a_{i}, b_{i}\right] \mid \lambda_{i} \in F, a_{i} \in A, b_{i} \in B, n \in N\right\} .
$$

(iii) A subspace $I$ of a Lie algebra $L$ is called an ideal if

$$
[x, y] \in I \text { for all } x \in L, y \in I \text {. }
$$


(iv) A Lie algebra $L$ is called commutative when $[x, y]=0$ for all $x, y \in L$.

(v) If $L_{1}$ and $L_{2}$ are Lie algebras over $F$, then we say that a map $\varphi: L_{1} \rightarrow L_{2}$ is a Lie homomorphism if $\varphi$ is a linear map and

$$
\varphi([x, y])=[\varphi(x), \varphi(y)] \text { forall } x, y \in L_{1} .
$$

Definition 2.7 Let $L$ and $L^{\prime}$ be two Lie algebras over field $F$ and $T: L \rightarrow P^{*}\left(L^{\prime}\right)$ be a set-valued mapping. $T$ is called a set-valued Lie homomorphism if

- $T(x+y)=T(x)+T(y)$

- $T(\lambda x)=\lambda T(x)$

- $\{[a, b] \mid a \in T(x), b \in T(y)\}=T[x, y]$.

for all $\lambda \in F$ and $x, y \in L$.

It is clear that $T(0)=\{0\}$ and $T(-x)=-T(x)$ for all $x \in L$.

Example 2.8 (i) Let $L$ is a Lie algebra and $T: L \rightarrow P^{*}\left(\frac{L}{I}\right)$ be a set-valued mapping and

$T(x)=\{x+I\}$ for all $x \in L$ which $I$ is an ideal of $L$. Then $T$ is a set-valued Lie homomorphism.

Here, $\frac{L}{I}$ is a Lie algebra over $F$ and its the Lie bracket defined by

$$
[x+I, y+I]=[x, y]+I \text { for all } x, y \in L \text {. }
$$

$\frac{L}{I}$ is called the quotient algebra of $L$ by $I$.

(ii) Let $f: L \rightarrow L^{\prime}$ be a Lie algebra homomorphism and $T: L \rightarrow P^{*}\left(L^{\prime}\right)$ defined by $T(x)=\{f(x)\}$, then $T$ is a set-valued Lie homomorphism.

(iii) Let $T: L \rightarrow P^{*}\left(L^{\prime}\right)$ be a set-valued mapping such that $T(x)=\{0\}$, then $T$ is a set-valued Lie homomorphism.

The following corollaries are clear.

Corollary 2.9 Let $T: L \rightarrow P^{*}\left(L^{\prime}\right)$ be a set-valued Lie homomorphism and $f: L^{\prime \prime} \rightarrow L$ be a Lie homomorphism, then Tof is a set-valued Lie homomorphism from $L^{\prime \prime}$ to $P^{*}\left(L^{\prime}\right)$ such that $U_{T o f}(B)=f^{-1}\left(U_{T}(B)\right)$ and $L_{T o f}(B)=f^{-1}\left(L_{T}(B)\right)$ for all $B \in P^{*}\left(L^{\prime}\right)$.

Corollary 2.10 Let $T: L \rightarrow P^{*}\left(L^{\prime}\right)$ be a set-valued Lie homomorphism and $f: L^{\prime} \rightarrow L^{\prime \prime}$ be a Lie homomorphism, then $T_{f}$ is a set-valued Lie homomorphism from $L$ to $P^{*}\left(L^{\prime \prime}\right)$ defined by $T_{f}(m)=f(T(m))$ such that $L_{T_{f}}(A)=L_{T}\left(f^{-1}(A)\right)$ and $U_{T_{f}}(A)=U_{T}\left(f^{-1}(A)\right)$ for all $A \in P^{*}\left(L^{\prime \prime}\right)$ and $m \in L$. 
Lemma 2.11 Let $W$ be an ideal $L^{\prime}$ and $T: L \rightarrow P^{*}\left(L^{\prime}\right)$ be a set-valued Lie homomorphism, then $L_{T}(W)$ and $U_{T}(W)$ are ideals of $L$.

Proof. It is clear that $0 \in L_{T}(W)$, therefore $L_{T}(W) \neq \varnothing$. Now we show that $L_{T}(W)$ is a subspace. Let $x, y \in L_{T}(W)$ and $\lambda \in F$, so $T(x) \subseteq W$ and $T(y) \subseteq W$. Therefore $\lambda T(x) \subseteq W$, hence $\lambda T(x)+T(y) \subseteq W$. Since $T$ is a Lie homomorphism, so $T(\lambda x)+T(y) \subseteq W$. It yields that $T(\lambda x+y) \subseteq W$. It shows that $\lambda x+y \in L_{T}(W)$. Now to show that $L_{T}(W)$ is an ideal, we need to check that $[x, y] \in L_{T}(W)$ for all $x \in L_{T}(W)$ and $y \in L$, or $T([x, y]) \subseteq W$. In fact we show that $\{[a, b] \mid a \in T(x), b \in T(y)\} \subseteq W$. Let $[u, v] \in\{[a, b] \mid a \in T(x), b \in T(y)\}$ since $u \in T(x) \subseteq W$, and $W$ is an ideal, then $[u, v] \in W$.

A little change to above proving, we can obtain the next result.

Lemma 2.12 Let $T: L \rightarrow P^{*}\left(L^{\prime}\right)$ be a set-valued Lie homomorphism, then $\operatorname{ker} T=\{x \in L \mid T(x)=\{0\}\}$ is an ideal of $L$.

Corollary 2.13 Let $T: L \rightarrow P^{*}\left(L^{\prime}\right)$ be a set-valued Lie homomorphism. If $A$ and $B$ be two non-empty sets of $L^{\prime}$, then $\left[U_{T}(A), U_{T}(B)\right] \subseteq U_{T}([A, B])$ and $\left[L_{T}(A), L_{T}(B)\right] \subseteq L_{T}([A, B])$.

The following example shows that in general, the converse of above relations do not hold:

Example 2.14 Let $L$ be a commutative Lie algebra and $A=L=B$ and $T: L \rightarrow P^{*}(L)$ defined by $T(x)=\{0\}$, then we have

(i) $L_{T}[A, B]=L_{T}[L, L]=L_{T}\{0\}=\{x \in L \mid T(x) \subseteq\{0\}\}=L$. On the other hand, $L_{T}(L)=\{x \in L \mid T(x) \subseteq L\}=L$, hence $\left[L_{T}(L), L_{T}(L)\right]=[L, L]=0$.

(ii) $U_{T}(L)=\{x \in L \mid T(x) \cap L \neq \varnothing\}=L$, hence $\left[U_{T}(L), U_{T}(L)\right]=[L, L]=0$. On the other hand, $U_{T}[L, L]=U_{T}\{0\}=\{x \in L \mid T(x) \cap\{0\} \neq \varnothing\}=L$.

Corollary 2.15 Let $T: L \rightarrow P^{*}\left(L^{\prime}\right)$ be a set-valued Lie homomorphism. If $A$ is a Lie subalgebra of $L^{\prime}$, then $U_{T}(A)$ and $L_{T}(A)$ are Lie subalgebras of $L$.

Proof. The proof is straightforward.

Theorem 2.16 Let $f: L \rightarrow L^{\prime}$ be an isomorphism and $T_{2}: L^{\prime} \rightarrow P^{*}\left(L^{\prime}\right)$ be a set-valued Lie homomorphism. If $T_{1}(x)=\left\{u \in L \mid f(u) \in T_{2}(f(x))\right\}$ for all $x \in L$, then $T_{1}$ is a set-valued Lie homomorphism from $L$ to $P^{*}(L)$.

Proof. First, we show that $T_{1}$ is a well-defined mapping. Suppose $x_{1}=x_{2}$, we have

$$
y_{1} \in T_{1}\left(x_{1}\right) \Leftrightarrow f\left(y_{1}\right) \in T_{2}\left(f\left(x_{1}\right)\right)=T_{2}\left(f\left(x_{2}\right)\right) \Leftrightarrow y_{1} \in T_{1}\left(x_{2}\right) .
$$


Then $T_{1}\left(x_{1}\right)=T_{1}\left(x_{2}\right)$. Now we show that $T_{1}\left(x_{1}+x_{2}\right)=T_{1}\left(x_{1}\right)+T_{1}\left(x_{2}\right)$.

Suppose $y \in T_{1}\left(x_{1}+x_{2}\right)$, then

$$
f(y) \in T_{2}\left(f\left(x_{1}+x_{2}\right)\right)=T_{2}\left(f\left(x_{1}\right)+f\left(x_{2}\right)\right)=T_{2}\left(f\left(x_{1}\right)\right)+T_{2}\left(f\left(x_{2}\right)\right) .
$$

Hence there exist $a \in T_{2}\left(f\left(\left(x_{1}\right)\right)\right.$ and $b \in T_{2}\left(f\left(\left(x_{2}\right)\right)\right.$ such that $f(y)=a+b$. Since $f$ is onto, then there exist $d, c \in L$ such that $f(c)=a, f(d)=b$. On the other hand, we have $f(c) \in T_{2}\left(f\left(x_{1}\right)\right)$, then $c \in T_{1}\left(x_{1}\right)$ and also $f(d) \in T_{2}\left(f\left(x_{2}\right)\right)$. Therefore $d \in T_{1}\left(x_{2}\right)$ and $f(y)=a+b=f(c)+f(d)=f(c+d)$. Since $f$ is one to one, it implies $y=c+d$. So $y \in T_{1}\left(x_{1}\right)+T_{1}\left(x_{2}\right)$. It follows $T_{1}\left(x_{1}+x_{2}\right) \subseteq T_{1}\left(x_{1}\right)+T_{1}\left(x_{2}\right)$.

Conversely, assume that $y \in T_{1}\left(x_{1}\right)+T_{1}\left(x_{2}\right)$, then there are $a \in T_{1}\left(x_{1}\right), b \in T_{1}\left(x_{2}\right)$ such that $y=a+b$. Hence

$$
\begin{aligned}
& f(y)=f(a)+f(b)=f(a+b) \in T_{2}\left(f\left(x_{1}\right)\right)+T_{2}\left(f\left(x_{2}\right)\right)=T_{2}\left(f\left(x_{1}+x_{2}\right)\right) . \\
& \quad \Rightarrow y \in T_{1}\left(x_{1}+x_{2}\right) .
\end{aligned}
$$

So $T_{1}\left(x_{1}\right)+T_{1}\left(x_{2}\right) \subseteq T_{1}\left(x_{1}+x_{2}\right)$. Also we show that $T_{1}(\lambda x)=\lambda T_{1}(x)$. Suppose $u \in T_{1}(\lambda x)$. So $f(u) \in T_{2}(f(\lambda x))=\lambda T_{2}(f(x))$. Then there exists $z \in T_{2}(f(x))$ such that $f(u)=\lambda z$. Since $f$ is onto, then there is $m \in L_{1}$ such that $z=f(m)$. Therefore we have $f(u)=\lambda f(m)=f(\lambda m)$ and since $f$ is one to one, it implies that $u=\lambda m$. So $z=f(m) \in T_{2}(f(x))$. It shows that $m \in T_{1}(x)$. Therefore $\lambda m \in \lambda T_{1}(x)$. Then $u \in \lambda T_{1}(x)$. Now for proving $\lambda T_{1}(x) \subseteq T_{1}(\lambda x)$, let $u \in \lambda T_{1}(x)$. By definition, there exists $z \in T_{1}(x)$ such that $u=\lambda z$. So $f(u)=\lambda f(z)$. Since $f(z) \in T_{2}(f(x))$ and $f(u) \in \lambda T_{2}(f(x))=T_{2}(f(\lambda x))$, hence $f(u) \in T_{2}(f(\lambda x)) \Rightarrow u \in T_{1}(\lambda x)$.

Now we show that $T_{1}$ preserves the Lie bracket, that means

$$
T_{1}[x, y]=\left\{[a, b] \mid a \in T_{1}(x), b \in T_{1}(y)\right\} .
$$

First, we show that $f[a, b] \in T_{2}(f[x, y])$ for all $a \in T_{1}(x)$ and $b \in T_{1}(y)$. Let $f(a) \in T_{2}(f(x))$ and $f(b) \in T_{2}(f(y))$. Since $f$ is a Lie homomorphism, therefore

$$
\begin{aligned}
f[a, b] & =[f(a), f(b)] \in\left\{[u, v] \mid u \in T_{2}(f(x)), v \in T_{2}(f(y))\right\} \\
& =T_{2}[f(x), f(y)] .
\end{aligned}
$$

It implies $\left\{[a, b] \mid a \in T_{1}(x), b \in T_{1}(y)\right\} \subseteq T_{1}[x, y]$. Now we show

$$
T_{1}[x, y] \subseteq\left\{[a, b] \mid a \in T_{1}(x), b \in T_{1}(y)\right\} .
$$

We have $T_{1}[x, y]=\left\{u \in L \mid f(u) \in T_{2}(f[x, y])\right\}$

$$
\begin{aligned}
& =\left\{u \in L \mid f(u) \in T_{2}([f(x), f(y)])\right\} \\
& =\left\{u \in L|f(u)|\left\{[a, b] \mid a \in T_{2}(f(x)), b \in T_{2}(f(y))\right\} .\right.
\end{aligned}
$$

Now let $f(u)=[a, b]$ then there exist $a \in T_{2}(f(x))$ and $b \in T_{2}(f(y))$. Since $f$ is onto, then there exist $d, c \in L$ such that $f(c)=a$ and $f(d)=b$. It is clear that $c \in T_{1}(x)$ and $d \in T_{1}(y)$ and $f(u)=[f(c), f(d)]=f[c, d]$. Since $f$ is one to one, then

$$
u=[c, d] \in\left\{\left[t_{1}, t_{2}\right] \mid t_{1} \in T_{1}(x), t_{2} \in T_{1}(y)\right\} \subseteq\left\{[a, b] \mid a \in T_{1}(x), b \in T_{1}(y)\right\} .
$$


Theorem 2.17 Let $f: L \rightarrow L^{\prime}$ be a Lie algebra isomorphism and let $T_{2}: L^{\prime} \rightarrow P^{*}\left(L^{\prime}\right)$ be a set-valued Lie homomorphism. If for any $x \in L, T_{1}(x)=\left\{u \in L \mid f(u) \in T_{2}(f(x))\right\}$ and $A$ is a subalgebra of $L^{\prime}$, then

(i) $f\left(L_{T_{1}}(A)\right)=L_{T_{2}}(f(A))$;

(ii) $f\left(U_{T_{1}}(A)\right)=U_{T_{2}}(f(A))$.

Proof. $(i)$ If $y \in f\left(L_{T_{1}}(A)\right)$, then there exists $x \in L_{T_{1}}(A)$ such that $y=f(x)$. But if $x \in L_{T_{1}}(A)$, then we have $T_{1}(x) \subseteq A$. Now if $w \in T_{2}(f(x))$, since $f$ is onto, then there exists $z \in L$ such that $w=f(z)$ . So,

$$
\begin{aligned}
& w=f(z) \in T_{2}(f(x)) \Rightarrow z \in T_{1}(x) \subseteq A \\
& \Rightarrow w=f(z) \in f(A) \\
& \Rightarrow T_{2}(f(x)) \subseteq f(A) \\
& \Rightarrow y \in L_{T_{2}}(f(A)) .
\end{aligned}
$$

Therefore $f\left(L_{T_{1}}(A)\right) \subseteq L_{T_{2}}(f(A))$.

Conversely, if $y \in L_{T_{2}}(f(A))$, then $T_{2}(y) \subseteq f(A)$. On the other hand, $f$ is onto, then there is $x \in L$ such that $y=f(x)$. Hence, we have $T_{2}(f(x)) \subseteq f(A)$.

Let $u \in T_{1}(x)$, then $f(u) \in f(A)$, therefore there exists $a \in A$ such that $f(u)=f(a)$. But $f$ is one to one, so $u=a$. Hence we have

$$
u \in A \Rightarrow T_{1}(x) \subseteq A \Rightarrow x \in L_{T_{1}}(A) \Rightarrow y \in f\left(L_{T_{1}}(A)\right) .
$$

So, $L_{T_{2}}(f(A)) \subseteq f\left(L_{T_{1}}(A)\right)$.

(ii) If $y \in f\left(U_{T_{1}}(A)\right)$, then there exists $x \in U_{T_{1}}(A)$ such that $y=f(x)$. But if $x \in U_{T_{1}}(A)$, then $T_{1}(x) \cap A \neq \varnothing$. Let $a \in T_{1}(x) \cap A$. Therefore

$$
\begin{aligned}
& f(a) \in T_{2}(f(x)) \cap f(A) \Rightarrow T_{2}(f(x)) \cap f(A) \neq \varnothing \\
& \Rightarrow f(x) \in U_{T_{2}}(f(A)) \\
& \Rightarrow y \in U_{T_{2}}(f(A)) .
\end{aligned}
$$

It means that $f\left(U_{T_{1}}(A)\right) \subseteq U_{T_{2}}(f(A))$. Conversely, if $y \in U_{T_{2}}(f(A))$, since $f$ is onto, then there exist $x \in L$ such that $y=f(x)$, and $T_{2}(y) \cap f(A) \neq \varnothing$. So, we have $T_{2}(f(x)) \cap f(A) \neq \varnothing$. Hence there is $z \in T_{2}(f(x)) \cap f(A)$. It means that there exists $a \in A$ such that $z=f(a) \in T_{2}(f(x))$. Then $a \in T_{1}(x) \cap A \neq \varnothing$. It obtains that $x \in U_{T_{1}}(A)$. Then $y=f(x) \in f\left(U_{T_{1}}(A)\right)$. It follows that $U_{T_{2}}(f(A)) \subseteq f\left(U\left(_{T_{1}}(A)\right)\right.$.

Definition 2.18 A congruence $\theta$ on $L$ is called complete if for any $x, y \in L$ and $r \in F$

(i) $[x]_{\theta}+[y]_{\theta}=[x+y]_{\theta}$;

(ii) $r[x]_{\theta}=[r x]_{\theta}$;

(iii) $[[x, y]]_{\theta}=\left\{[a, b] \mid a \in[x]_{\theta}, b \in[y]_{\theta}\right\}$. 
By using the above theorems and definition, we obtain the following:

Corollary 2.19 Let $\theta_{2}$ be a complete congruence relation on Lie algebra $L_{2}$ and $f: L_{1} \rightarrow L_{2}$ be a Lie algebra isomorphism and $\theta_{1}=\left\{(x, y) \in L_{1} \times L_{1} \mid f(x), f(y) \in \theta_{2}\right\}$, then $\theta_{1}$ is a complete congruence relation on $L_{1}$ that $\varnothing \neq A \subseteq L_{1}$

(i) $f\left(\underline{A p r}_{\theta_{1}}(A)\right)=\underline{A p r}_{\theta_{2}}(f(A))$;

(ii) $f\left(\overline{\operatorname{Apr}}_{\theta_{1}}(A)\right)=\overline{\operatorname{Apr}}_{\theta_{2}}(f(A))$.

\section{Generalized $T$-rough Lie algebras}

In this section, we define a $T$-rough Lie algebra with respect to a Lie subalgebra of a Lie algebra, is called the generalized $T$-rough Lie algebra and study some of their appealing properties.

Definition 3.1 Let $A$ and $B$ be two Lie subalgebras of $L^{\prime}$ and $T: L \rightarrow P^{*}\left(L^{\prime}\right)$ be a set-valued Lie homomorphism, then

$$
L_{T}^{A}(B)=\{x \in L \mid(T(x)+A) \subseteq B\} ; U_{T}^{A}(B)=\{x \in L \mid(T(x)+A) \cap B \neq \varnothing\}
$$

are called the generalized lower and upper approximations of $B$, respectively, with respect to the Lie subalgebra $A$.

In the special case, if $A=0$, then $L_{T}^{A}(B)=L_{T}(B)$ and $U_{T}^{A}(B)=U_{T}(B)$. Furthermore, if $0 \in A \subseteq B$, then $L_{T}^{A}(B) \subseteq L_{T}(B)$ and $U_{T}^{A}(B) \subseteq U_{T}(B)$.

Definition 3.2 Let $L$ be a Lie algebra. If $A$ be a Lie subalgebra of $L$ and $\varnothing \neq S \subseteq F$, then $S A$ denotes the set of $\left\{\sum_{i=1}^{n} s_{i} a_{i} \mid s_{i} \in S, a_{i} \in A, n \in \mathbf{N}\right\}$.

Theorem 3.3 Let $T: L \rightarrow P^{*}\left(L^{\prime}\right)$ be a set-valued Lie homomorphism, the following holds:

(i) If $A$ and $B$ are two Lie subalgebras of $L^{\prime}$ such that $A \subseteq B$, then $L_{T}^{A}(B)$ is Lie subalgebra of $L$;

(ii) If $A$ is an ideal of $L^{\prime}$ and $B$ is a Lie subalgebra of $L^{\prime}$, then $U_{T}^{A}(B)$ is Lie subalgebra of $L$.

Proof. $(i)$ First, we show that $L_{T}^{A}(B)$ is a subspace. It is clear that $0 \in L_{T}^{A}(B)$. Now suppose that $x, y$ be two elements of $L_{T}^{A}(B)$ and $\lambda \in F$. If $x \in L_{T}^{A}(B)$, since $A$ is a Lie subalgebra, hence $0 \in A$ then $T(x) \subseteq B$, so $\lambda T(x) \subseteq B$. On the other hand, $A \subseteq B$. Therefore $\lambda T(x)+A \subseteq B$, and so $T(\lambda x)+A \subseteq B$. Also $T(y)+A \subseteq B$. Hence $T(\lambda x)+T(y)+A \subseteq B$. It deduces that $T(\lambda x+y)+A \subseteq B$. Therefore $\lambda x+y \in L_{T}^{A}(B)$. Now we show that if $x, y \in L_{T}^{A}(B)$, then $[x, y] \in L_{T}^{A}(B)$. Since $T[x, y]=\{[a, b] \mid a \in T(x), b \in T(y)\}$, from $a \in T(x)$ and $b \in T(y)$, we have 
$a \in B$ and $b \in B$, therefore $[a, b] \in B$. It means that $\{[a, b] \mid a \in T(x), b \in T(y)\} \subset B$, so $T[x, y] \subset B$ . On the other hand, $A \subseteq B$ which implies $T[x, y]+A \subseteq B$. It follows $[x, y] \in L_{T}^{A}(B)$.

(ii) First, we show that $U_{T}^{A}(B)$ is a subspace. It is clear that $0 \in U_{T}^{A}(B)$. Now suppose that $x, y$ be two elements of $U_{T}^{A}(B)$ and $\lambda \in F$. If $x \in U_{T}^{A}(B) \Rightarrow(T(x)+A) \cap B \neq \varnothing$ and since $y \in U_{T}^{A}(B)$, then $(T(y)+A) \cap B \neq \varnothing$, so there exist $a, b \in L^{\prime}$ such that $a \in(T(x)+A) \cap B$ and $b \in(T(y)+A) \cap B$. It implies that $\lambda a \in T(\lambda x)+\lambda A \subseteq T(\lambda x)+A$. On the other hand, $\lambda a \in \lambda B \subset B$, so $\lambda a \in B$. It deduces that $\lambda a \in(T(\lambda x)+A) \cap B$, since $b \in(T(y)+A) \cap B$, therefore $\lambda a+b \in(T(\lambda x+y)+A) \cap B$. It shows that $(T(\lambda x+y)+A) \cap B \neq \varnothing$. It follows $\lambda x+y \in U_{T}^{A}(B)$.

Now we show that if $x, y \in U_{T}^{A}(B)$, then $[x, y] \in U_{T}^{A}(B)$. Since $x \in U_{T}^{A}(B)$, then $(T(x)+A) \cap B \neq \varnothing, \quad$ so there exists $u \in(T(x)+A) \cap B$, and since $y \in U_{T}^{A}(B)$, then $(T(y)+A) \cap B \neq \varnothing$ so there exists $v \in(T(x)+A) \cap B$. It implies that $[u, v] \in B$. Let $u=x_{1}+a_{1}$ such that $x_{1} \in T(x), a_{1} \in A$ and $v=x_{2}+a_{2}$ such that $x_{2} \in T(y), a_{2} \in A$, we have

$$
\begin{aligned}
{[u, v] } & =\left[x_{1}+a_{1}, x_{2}+a_{2}\right] \\
& =\left[x_{1}, x_{2}\right]+\left[x_{1}, a_{2}\right]+\left[a_{1}, x_{2}\right]+\left[a_{1}, a_{2}\right] \in B .
\end{aligned}
$$

Since $A$ is ideal of Lie algebra $L^{\prime}$, so $\left[x_{1}, a_{2}\right],\left[a_{1}, x_{2}\right],\left[a_{1}, a_{2}\right] \in A$. Hence we have

$$
\begin{aligned}
\left\{\left[x_{1}, x_{2}\right]+A \mid x_{1} \in T(x), x_{2} \in T(y)\right\} \subset B & \Rightarrow T[x, y]+A \subset B \\
& \Rightarrow T[x, y]+A \cap B \neq \varnothing \\
& \Rightarrow[x, y] \in U_{T}^{A}(B) .
\end{aligned}
$$

Notice: In the above theorem, the condition $A \subseteq B$ is necessary, because $0 \notin L_{T}^{A}(B)$.

The following example shows that in condition ( $i i)$ to $A$ 's being ideal is a necessity.

Example 3.4 Let $L_{1}=L_{2}=g l(n, F)$ be the set of all $n \times n$ matrices over $F$ and

$T: g l(n, F) \rightarrow P^{*}(g l(n, F))$ and for any $x \in g l(n, F)$ we define $T(x)=\{x\}$. Now if $A=b(n, F)$ is the upper triangular matrices and $B=\{0\}$, then $A$ is not an ideal of $g l(n, F)$ and $U_{T}^{A}(B)=A$. for

$$
\begin{aligned}
U_{T}^{A}(B) & =\{x \in L \mid(T(x)+A) \cap B \neq \varnothing\} \\
& =\{x \in L \mid\{x\}+b(n, F) \cap\{0\} \neq \varnothing\}=b(n, F)=A .
\end{aligned}
$$

Now we have $T$ is a Lie algebra homomorphism

(i) $T(A+B)=\{A+B\}=\{A\}+\{B\}=T(A)+T(B)$;

(ii) $T(\lambda A)=\{\lambda A\}=\lambda\{A\}=\lambda T(A)$;

(iii) $T[A, B]=\{[A, B]\}=\{[a, b] \mid a \in T(A), b \in T(B)\}$.

Lemma 3.5 Let $L$ be a Lie algebra and $A, B$ be non-empty Lie subalgebras of $L^{\prime}$ such that $A \subseteq B$ and let $S$ be a subspace of $L^{\prime}$. If $T: L \rightarrow P^{*}\left(L^{\prime}\right)$ be a set-valued Lie homomorphism, then

(i) $L_{T}^{B}(S) \subseteq L_{T}^{A}(S)$; 
(ii) $U_{T}^{A}(S) \subseteq U_{T}^{B}(S)$.

Proof. The proof is straightforward.

The following corollary follows by Lemma 3.5.

Corollary 3.6 Let $A, B$ be Lie subalgebras of $L^{\prime}$ and $S$ be a non-empty subset of $L^{\prime}$. If

$T: L \rightarrow P^{*}\left(L^{\prime}\right)$ be a set-valued Lie homomorphism, then

(i) $L_{T}^{A}(S) \cap L_{T}^{B}(S) \subseteq L_{T}^{A \cap B}(S)$;

(ii) $U_{T}^{A \cap B}(S) \subseteq U_{T}^{A}(S) \cap U_{T}^{B}(S)$.

Theorem 3.7 Suppose $S$ be a non-empty subset of $F$ and $B$ be Lie subalgebra of $L$ and $A$ be a subspace of $L^{\prime}$. If $T: L \rightarrow P^{*}\left(L^{\prime}\right)$ be a set-valued Lie homomorphism, then

(i) If $A \subseteq B$, then $S L_{T}^{A}(B) \subseteq L_{T}^{A}(S B)$;

(ii) $S U_{T}^{A}(B) \subseteq U_{T}^{A}(S B)$.

Proof. (i) Let $x$ be any element of $S L_{T}^{A}(B)$, then $x=\sum_{i=1}^{n} s_{i} b_{i}$ for some $s_{i} \in S, b_{i} \in L_{T}^{A}(B)$ and $n \in \mathbf{N}$. Now, we have $T\left(b_{i}\right)+A \subseteq B$, and so $s_{i} T\left(b_{i}\right)+A \subseteq s_{i} B \subseteq S B$, for all $i=1,2, \ldots, n$. Then we have $T\left(s_{i} b_{i}\right)+A \subseteq S B \quad$ which implies $\quad s_{i} b_{i} \in L_{T}^{A}(S B)$. Therefore $\quad x=\sum_{i=1}^{n} s_{i} b_{i} \in L_{T}^{A}(S B)$ and $\quad$ so $S L_{T}^{A}(B) \subseteq L_{T}^{A}(S B)$.

(ii) Let $x$ be any element of $S U_{T}^{A}(B)$, then $x=\sum_{i=1}^{n} s_{i} b_{i}$ for some $s_{i} \in S, b_{i} \in U_{T}^{A}(B)$ and $n \in \mathbf{N}$. Now, we have $\left(T\left(b_{i}\right)+A\right) \cap B \neq \varnothing$ for all $i=1,2, \ldots, n$. So there exists $a_{i} \in\left(T\left(b_{i}\right)+A\right) \cap B$. Hence $s_{i} a_{i} \in B \quad$ and $\quad s_{i} a_{i} \in s_{i} T\left(b_{i}\right)+A=T\left(s_{i} b_{i}\right)+A$. So $\quad \sum_{i=1}^{n} s_{i} a_{i} \in S B \quad$ and $\quad \sum_{i=1}^{n} s_{i} a_{i} \in T\left(\sum_{i=1}^{n} s_{i} b_{i}\right)+A$. Therefore $\Sigma_{i=1}^{n} s_{i} a_{i} \in(T(x)+A) \cap S B$. Thus $(T(x)+A) \cap S B \neq \varnothing$ which implies $x \in U_{T}^{A}(S B)$, and so $S U_{T}^{A}(B) \subseteq U_{T}^{A}(S B)$.

Theorem 3.8 Suppose that $A, B$ and $C$ be Lie subalgebras of $L^{\prime}$. If $T: L \rightarrow P^{*}\left(L^{\prime}\right)$ be a set-valued Lie homomorphism, then

(i) $L_{T}^{A}(C)+L_{T}^{B}(C)=L_{T}^{A+B}(C)$;

(ii) $U_{T}^{A}(C)+U_{T}^{B}(C)=U_{T}^{A+B}(C)$.

Proof. (i) Since $A \subseteq A+B$ and $B \subseteq A+B$, then by Lemma 3.5,

$$
L_{T}^{A+B}(C) \subseteq L_{T}^{A}(C) \subseteq L_{T}^{A}(C)+L_{T}^{B}(C)
$$


Now, let $x \in L_{T}^{A}(C)+L_{T}^{B}(C)$, then $x=y+z$ for some $y \in L_{T}^{A}(C)$ and $z \in L_{T}^{B}(C)$. Hence $T(y)+A \subseteq C$ and $T(z)+B \subseteq C$, then $T(y+z)+A+B \subseteq C$ which implies $x \in L_{T}^{A+B}(C)$. Therefore we obtain $L_{T}^{A}(C)+L_{T}^{B}(C)=L_{T}^{A+B}(C)$.

(ii) Since $A \subseteq A+B$ and $B \subseteq A+B$, by Lemma 3.5, $U_{T}^{A}(C) \subseteq U_{T}^{A+B}(C)$ and $U_{T}^{B}(C) \subseteq U_{T}^{A+B}(C)$ and so $U_{T}^{A}(C)+U_{T}^{B}(C) \subseteq U_{T}^{A+B}(C)$. Also $U_{T}^{A+B}(C) \subseteq U_{T}^{A}(C) \subseteq U_{T}^{A}(C)+U_{T}^{B}(C)$.

And the equality in relation (ii) is true when $A+B$ is an ideal. Now we have $U_{T}^{A+B}(C) \subseteq U_{T}^{A}(C)+U_{T}^{B}(C)$. Let $x \in U_{T}^{A+B}(C)$, then $(T(x)+A+B) \cap C \neq \varnothing$, so there exists $u \in T(x)+A+B \cap C$, therefore we have $u=x_{1}+a+b$ such that $x_{1} \in T(x), a \in A, b \in B$, so $u-b=x_{1}+a \in T(x)+A$. Since $A+B$ is an ideal, then $U_{T}^{A+B}(C)$ is a Lie subalgebra, so $A+B \subset C$. On the other hand, $A \subset A+B \subset C$ and $B \subset A+B \subset C$. Hence $A \subset C$ and $B \subset C$. So

$$
(T(x)+A) \cap C \neq \varnothing \Rightarrow x \in U_{T}^{A}(C) \subset U_{T}^{A}(C)+U_{T}^{B}(C) .
$$

Proposition 3.9 Let $L$ be a Lie algebra and $A$ is a Lie subalgebra of $L^{\prime}$ and $B$ is non-empty subset of L', then

(i) $L_{T}^{A}\left(B^{c}\right)=\left(U_{T}^{A}(B)\right)^{c}$;

(ii) $U_{T}^{A}\left(B^{c}\right)=\left(L_{T}^{A}(B)\right)^{c}$.

Proof. (i) We have

$$
\begin{aligned}
& x \in L_{T}^{A}\left(B^{c}\right) \Leftrightarrow T(x)+A \subseteq B^{c} \Leftrightarrow(T(x)+A) \cap B=\varnothing \\
& \Leftrightarrow x \notin U_{T}^{A}(B) \Leftrightarrow x \in\left(U_{T}^{A}(B)\right)^{c} .
\end{aligned}
$$

(ii) By substitution $B^{c}$ for $B$ in $(i)$ we get $U_{T}^{A}\left(B^{c}\right)=\left(L_{T}^{A}(B)\right)^{c}$.

\section{Conclusion}

In this work, the lower $T$-rough and upper $T$-rough Lie subalgebras are formulated in the context of Lie algebra theory. We introduced the notion of the set-valued Lie homomorphism and generalized $T$-rough Lie subalgebra in a Lie algebra which is an extended notion of Lie homomorphism and Lie subalgebra of a Lie algebra. We hope that this extended research may provide a powerful tool in approximate reasoning. We strongly believe that $T$-rough Lie algebra offered here will turn out to be more useful in the theory and applications of the rough sets.

\section{Acknowledgement}

The authors are highly grateful to an anonymous for her/his valuable comments and suggestions for improving the paper.

\section{References}

[1] R. Biswas, S. Nanda, Rough groups and rough subgroups, Bull. Polish Acad. Sci. Math 42(1994), 251-254.

[2] Z. Bonikowaski, Algebraic structures of rough sets, in: W.P. Ziarko (Ed.), Rough Sets, Fuzzy Sets and Knowledge Discovery, Springer-Verlag, Berlin, 1995, pp. 242-247. 
S. B. Hosseini, A. Kazemi / J. Math. Computer Sci. 14 (2014), 288-299

[3] B. Davvaz, A short note on algebraic T -rough sets, Information Sciences 178 (2008) 3247-3252.

[4] B. Davvaz, Roughness in rings, Information Sciences 164 (2004), 147-163.

[5] B. Davvaz, M. Mahdavipour, Roughness in modules, Information Sciences 176 (2006) 3658-3674.

[6] S. B. Hosseini, N. Jafarzadeh, A. Gholami, $T$-rough Ideal and $T$-rough Fuzzy Ideal in a Semigroup, Advanced Materials Research, Vols. 433-440 (2012) pp 4915-4919.

[7] S. B. Hosseini, N. Jafarzadeh, A. Gholami, Some Results on $T$-rough (prime, primary) Ideal and $T$-rough Fuzzy (prime, primary) Ideal on Commutative Rings, Int. J. Contemp. Math. Sciences, Vol. 7, 2012, no. 7, 337 - 350

[8] T. Iwinski, Algebraic approach to rough sets, Bull. Polish Acad. Sci. Math. 35 (1987), 673-683.

[9] O. Kazanci, B. Davvaz, On the structure of rough prime (primary) ideals and rough fuzzy prime (primary) ideals in commutative rings, Information Sciences, 178 (2008), 1343-1354.

[10] N. Kuroki, Rough ideals in semigroups, Information Sciences ,100 (1997), 139-163.

[11] Z. Pawlak, Rough sets basic notions, ICS PAS Rep. 436(1981).

[12] Z. Pawlak, Rough sets, Int. J. Inform. Comput. Sci. 11 (1982),341-356.

[13] Z. Pawlak, A. Skowron, Rough sets: some extensions, Information Sciences, 177 (2007), 28-40.

[14] W. Zhang, W. Wu, Theory and Method of Roughness, Science Press, Beijing, 2001. 